\title{
Tera International Unit Per Liter
}

National Cancer Institute

\section{Source}

National Cancer Institute. Tera International Unit Per Liter. NCI Thesaurus. Code

C105518.

Unit of arbitrary substance concentration (biologic activity concentration) defined as the concentration of $10^{\wedge} 12$ international unit per one liter of system volume. 